\title{
Ecophysiological Changes in a Cold Tolerant Transgenic Tobacco Plant Containing a Zinc Finger Protein (PIF1) Gene
}

\author{
Sung-Chul Yun* and Hawk-Bin Kwon \\ Department of Biomedical Sciences, Sun Moon University, Asan, 336-708, Korea \\ (Received September 18, 2008, Accepted December 12, 2008)
}

\begin{abstract}
The ecophysiological changes occurring upon cold stress were studied using cold tolerant transgenic and wild-type tobacco plants. In a previous study, cold tolerance in tobacco was induced by the introduction of a gene encoding the zinc finger transcription factor, PIF1. Gas-exchange measurements including net photosynthesis and stomatal conductance were performed prior to, in the middle of, and after a cold-stress treatment of $1 \pm 2{ }^{\circ} \mathrm{C}$ for $96 \mathrm{~h}$ in each of the four seasons. In both transgenic and wild-type plants, gas-exchange parameters were severely decreased in the middle of the cold treatment, but had recovered after 2-3 h of adaptation in a greenhouse. Most t-test comparisons on gas-exchange measurements between the two plant types did not show statistical significance. Wild-type plants had slightly more water-soaked damage on the leaves than the transgenic plants. A light-response curve did not show any differences between the two plant types. However, the curve for assimilation-intemal $\mathrm{CO}_{2}$ in wild-type plants showed a much higher slope than that of the PIF1 transgenic plants. This means that the wild-type plant is more capable of regenerating Ribulose-1,5-bisphosphate carboxylase/oxygenase (Rubisco) and has greater electron transport capacity. In conclusion, cold-resistant transgenic tobacco plants demonstrated a better recovery of net photosynthesis and stomatal conductance after cold-stress treatment compared to wild-type plants, but the ecophysiological recoveries of the transgenic plants were not statistically significant.
\end{abstract}

Key Words: cold-stress, zinc finger protein, transgenic tobacco, net photosynthesis, stomatal conductance

\section{INTRODUCTION}

Abiotic stresses including extreme temperature changes, drought, and high salinity cause major problems in agriculture by reducing crop yields. Because plants are sessile, they have evolved diverse mechanisms to cope with adverse environmental stress. One of the most important approaches is that plants turn on various stress-responsive genes that lead to changes at the biochemical and physiological level. The products of these stress responsive genes are involved in physiological defense or signal transduction pathways or regulation of expression of other stress responsive genes ${ }^{1)}$. A variety of stress responsive genes have been introduced into many plants to develop stress-

*Corresponding author:

Tel: +82-41-530-2282 Fax: +82-41-530-2939

E-mail: scyun@sunmoon.ac.kr resistant transgenic plants. These include Arabidopsis ${ }^{2,3)}$, soybean, and tobacco plants ${ }^{4,5)}$.

Previously, in an effort to elucidate a plant's mechanism of coping with cold stress, we isolated 317 cold-responsive genes from Capsicum annuиm using cDNA microarray and Northern blot analyses ${ }^{6}$. Among these genes, we selected a gene encoding a transcription factor, zinc finger protein (PIF1), and introduced it into tobacco plants ${ }^{7}$. We showed that the resulting PIF1 transgenic tobacco plants were strongly cold tolerant, but not drought tolerant ${ }^{7}$. However, in those studies, PIF1 transgenic tobacco treated with $-6^{\circ} \mathrm{C}$ cold stress could survive while wild-type plants could not. That result led to an examination of a variety of ecophysiological changes during cold stress as well as the stages of recovery after stress treatment.

Thus, in this study, we tried to verify that 
overexpression of the PIF1 gene in cold-tolerant transgenic tobacco plants could cause changes in ecophysiology including net photosynthesis (Pnet) and stomatal conductance (Cs). If transcriptional control by PIF1 leads to tolerance of cold stress by changing physiological functions, then there is a possibility that PIF1 may control Cs and other cellular physiological changes. Furthermore, the mechanism of cold-stress tolerance caused by the introduction of PIF1 could be explained at the ecophysiological level.

In addition, we performed a comparison of ecophysiological changes between PIF1 transgenic and wild-type tobacco plants throughout four seasons, as external temperature changes could have adverse affects on the measurement of gas exchange. By changing the light intensity of the chamber that measures gas-exchange ability (light curve) or changing the $\mathrm{CO}_{2}$ concentration (assimilation-internal $\mathrm{CO}_{2}$ curve: A-Ci curve), we could investigate differences in ecophysiological characteristics between cold-tolerant PIF1 transgenic and wild-type tobacco plants more thoroughly.

Here we report ecophysiological differences between cold-tolerant PIF1 transgenic and wild-type tobacco plants. We compared the gas-exchange abilities of both plant types during the stress treatment and recovery stages. In addition, we compared light curves and A-Ci curves of both plant types growing under normal conditions.

\section{Materials and Methods}

\section{Plant materials}

Tobacco plants (Nicotiana tabacum var. Xanti) were grown on MS culture media (MS salt, vitamin mix, $3 \%$ sucrose, $0.7 \%$ phytoagar, $\mathrm{pH} 5.7$ ) in a tissue culture room at $23^{\circ} \mathrm{C}$ under continuous light. The PIF1 transgenic tobacco plants were generated using Agrobacterium-mediated transformation methods for tobacco leaves ${ }^{7}$. Non-transgenic wild-type control plants were prepared for the identical analysis of gas exchange under the same culture conditions as those of the PIF1 transgenic tobacco plants.

Plants in culture media were grown until they had fully extended roots and 4-5 main leaves had appeared. These plants were acclimated in Jiffy pots for a week and then transferred to soil. After one week, the plants were used for cold-stress treatment.
Cold-stress treatment and examination of visible damage

Three different lines each of wild-type and PIF1 transgenic tobacco plants in pots were prepared for gas-exchange measurements following cold-stress treatment. For cold-stress treatment, the plants were kept in a $1 \pm 2^{\circ} \mathrm{C}$ refrigerator for $96 \mathrm{~h}$. Before measuring gas-exchange ability at each stage, the extent of water-soaked and wilting symptoms on the leaves, the typical visible symptoms of cold-stress damage, was observed. Although cold-stress treatment and gas-exchange measurements were conducted in the controlled environments of a greenhouse, in consideration of seasonal effects, we conducted measurements throughout the four seasons, i.e., late fall (November 2007), midwinter (February 2008), late spring (June 2008), and midsummer (August 2008).

\section{Gas-exchange measurements}

For measurements of Pnet and Cs, a Li-Cor 6400 photosynthesis measurement system (Li-Cor, Lincoln, $\mathrm{NE)}$ was used to measure gas-exchange ability. Gas-exchange measurements were performed at three stages; before treatment, after $48 \mathrm{~h}$, and $96 \mathrm{~h}$ after $1 \pm 2^{\circ} \mathrm{C}$ cold treatment. The 96-h cold-stress-treated plants were allowed to recover for $2-3 \mathrm{~h}$ in the greenhouse before measuring their gas-exchange ability.

Leaves were measured in $2 \mathrm{~cm} \times 3 \mathrm{~cm}$ photosynthesis measuring cuvettes under $1500 \mathrm{~mol} \mathrm{~m}^{-2} \mathrm{~s}^{-1}$ light emitting diode (LED) light, $400 \mathrm{mmol} \mathrm{L}^{-1} \mathrm{CO}_{2}$ and a cuvette temperature of $25^{\circ} \mathrm{C}$. Measurements were conducted using the youngest among the 4-5 fully expanded leaves of the wild-type and PIF1 transgenic plants.

A liquid $\mathrm{CO}_{2}$ supply system (Li-Cor 6400-01, Lincoln, $\mathrm{NE}$ ) attached to the photosynthesis measurement system was used to measure photosynthetic changes based on changes in supplied $\mathrm{CO}_{2}$ concentration. $\mathrm{CO}_{2}$ concentration was adjusted to 400, 300, 200, 100, 50, and $0 \mathrm{mmol} \mathrm{L} \mathrm{L}^{-1} \mathrm{CO}_{2}$. The measuring cuvette conditions were $1500 \mathrm{~mol} \mathrm{~m}^{-2} \mathrm{~s}^{-1}$ photosynthetically active radiation (PAR), $25^{\circ} \mathrm{C}$, and $30-60 \%$ relative humidity. To measure photosynthetic ability at different light intensities, the light saturation and compensation points of the two types of plants were measured with decreasing light intensity at 2000, 1500, 1000, 500, 200, $100,50,20,0 \mathrm{~mol} \mathrm{~m}^{-2} \mathrm{~s}^{-1}$. 


\section{Statistical analysis}

To examine the gas-exchange abilities of the two types of tobacco plants before, in the middle of, and after the cold-stress treatments, a one-tailed t-test was performed. Based on an alternative hypothesis that PIF1 transgenic plants would be more tolerant to cold stress than wild-type plants, we judged whether PIF1 transgenic plants would have higher Pnet and Cs than wild-type plants. We decided on a 95\% significance level, and used the statistics software program S-link (ver. 2.2, Seoul, Korea).

\section{Results and Discussion}

Visible damage on the two types of plants under cold-stress treatment

Leaves of wild-type plants developed weak watersoaked lesions and wilting symptoms after 48 and $96 \mathrm{~h}$ of $1 \pm 2^{\circ} \mathrm{C}$ cold treatment. As the external temperature was lower than that in the cold refrigerator during the February 2008 experiment, leaves of both types of plants showed water-soaked lesions and wilting symptoms; thus, we could not measure Pnet in the middle of the cold-stress treatment.

Previously, we reported that $-6^{\circ} \mathrm{C}$ treatment for 24 $h$ caused dramatic phenotypic differences between wild-type and PIF1 transgenic plants ${ }^{7}$. However, in this experiment $1 \pm 2^{\circ} \mathrm{C}$ cold treatment did not cause any significant visible difference between the two types of plants.

\section{Comparison of gas-exchange ability during cold-stress} treatment

The results of photosynthetic measurements of both types of plants throughout the four seasons revealed that Pnet in pre-cold treatment plants showed seasonal differences of 4.4 19.9 $\mu \mathrm{mol} \mathrm{CO} 2 \mathrm{~m}^{-2} \mathrm{~s}^{-1}$ (Fig. 1). The photosynthetic rate was lowest in February 2008 and transgenic plants showed a slightly higher Pnet compared to wild-type plants. However, in other experiments, During the pre-cold treatment, Pnet of wild-type plants was slightly higher than that of PIF1 transgenic plants. When measured within 5-10 min of the end of 48 -h of cold treatment, PIF1 transgenic plants showed a significantly $(p=0.05)$ higher Pnet than wild-type plants, but only in November 2007.

When the photosynthetic rate was measured in plants that had a 2-3-h recovery period in a greenhouse after completion of $96 \mathrm{~h}$ of cold treatment, PIF1 transgenic plants showed a tendency toward a higher Pnet throughout the four seasons as measured in August $2008(\mathrm{p}=0.05)$ and in November and February 2008 ( $\mathrm{p}=0.055$ and $\mathrm{p}=0.063$, respectively (Fig. 1)). Except for the measurement in August 2008, the 96-h

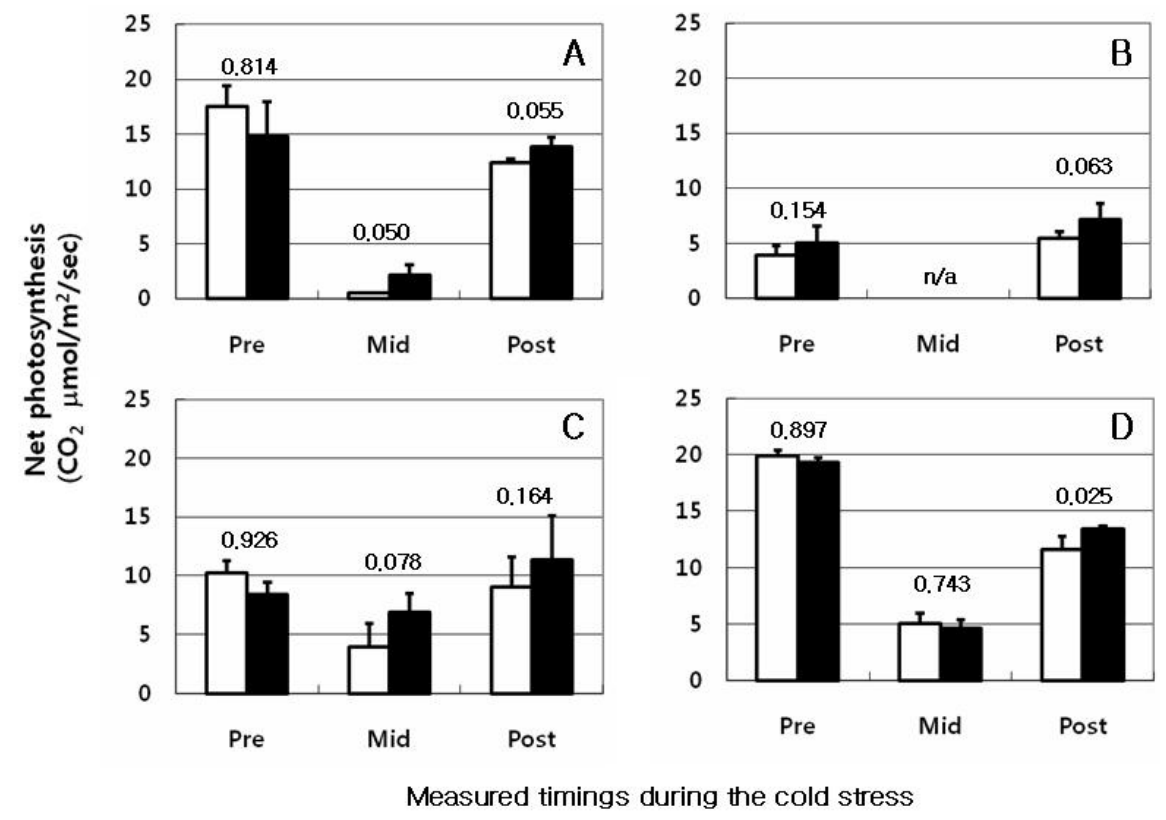

Fig. 1. Net photosynthesis in wild-type $(\square)$ and cold-stress tolerant ( $\square$ ) tobacco pre-treatment, mid-treatment, and on the last day of cold-stress treatment. The measurements were conducted in November 2007 (A), February 2008 (B), June 2008 (C), and August 2008 (D). The numbers on the bars are the p-values of $t$-tests between the two plant types. 
cold treatment followed by a 2-3-h recovery period restored the photosynthetic rate to that of the pre-cold treatment. A 2-3-h recovery period could also restore most of the water-soaked lesions and wilting symptoms on leaves to normal conditions.

The results of the Cs measurements throughout the four seasons revealed a pattern similar to that observed for the Pnet of both types of plants in that the pre-cold treatment showed seasonal differences of 0.03 0.6 $\mathrm{mmol} \mathrm{H}_{2} \mathrm{O} \mathrm{m}^{-2} \mathrm{~s}^{-1}$ (Fig. 2). Wild-type plants showed higher $\mathrm{Cs}$ during pre-treatment than PIF1 transgenic plants. However, the Cs results after 48 and $96 \mathrm{~h}$ of cold treatment followed by recovery revealed that the PIF1 transgenic plants showed a tendency toward a higher conductance compared to the wild-type plants. The differences between wild-type and PIF1 transgenic plants were significant in the February 2008 experiment $(\mathrm{p}=0.002)$, but not in the other seasons ( $\mathrm{p}=0.085 \sim 0.414$ based on one-tailed t-tests; Fig. 2).

Because the temperature of the greenhouse in fall and winter affected gas-exchange measurements, we could not measure gas exchange during the middle of the cold treatment. Pnet was measured as $4 \sim 8 \mu \mathrm{mol}$ $\mathrm{CO}_{2} \mathrm{~m}^{-2} \mathrm{~s}^{-1}$ and $\mathrm{Cs}$ as $0.01 \sim 0.02 \mathrm{mmol} \mathrm{H}_{2} \mathrm{O} \mathrm{m} \mathrm{m}^{-2} \mathrm{~s}^{-1}$ during the experiments performed in late spring and midsummer. The PIF1 transgenic plants showed a tendency toward higher Pnet and Cs values in the middle of and after cold treatment compared to wild-type plants, although in many cases the difference was not statistically significant.

Although there were seasonal differences, the mean Pnet and Cs of PIF1 transgenic plants were 10 30\% (Fig. 1) and 30 75\% (Fig. 2), respectively, higher than those observed for wild-type plants. This suggests that PIF1 zinc finger protein expression induces a series of proteins that protect plants from cold exposure, and these proteins may cause PIF1 transgenic plants to maintain a high Cs under cold temperatures, which may in turn contribute to an increase in the Pnet of PIF1 transgenic plants.

\section{Ecophysiological characteristics of cold-tolerant transgenic and wild-type tobacco plants}

To observe intrinsic ecophysiological characteristics of cold-tolerant transgenic and wild-type tobacco plants, we measured the $\mathrm{A}-\mathrm{Ci}$ and light response curves for both plant types. The A-Ci curve, which measures Pnet at each step as the $\mathrm{CO}_{2}$ concentration decreases in the cuvette, is restricted at lower $\mathrm{CO}_{2}$ concentrations due to the limited carboxylation activity of Ribulose-1,5-bisphosphate carboxylase/oxygenase (Rubisco).

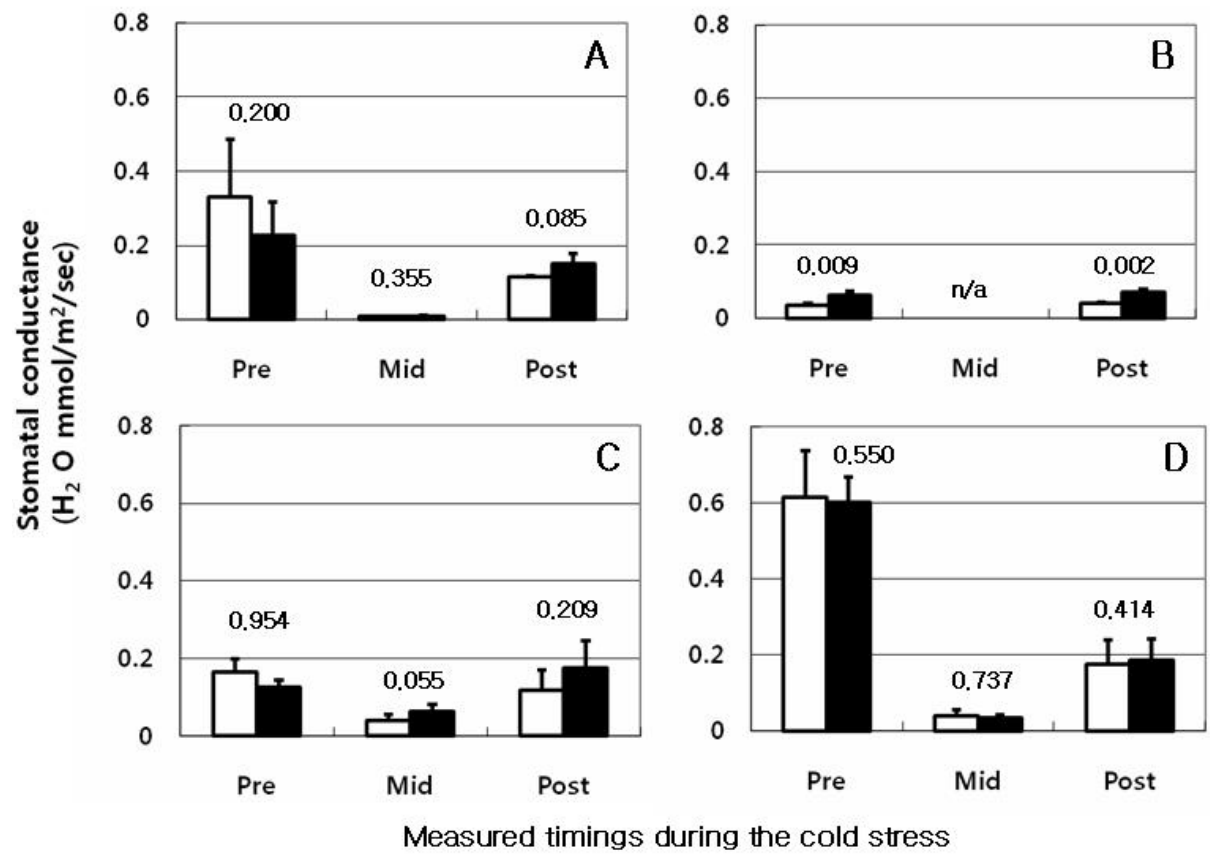

Fig. 2. Stomatal conductance in wild-type $(\square)$ and cold-stress tolerant $(\square)$ tobacco pre-treatment, mid-treatment, and on the last day of cold-stress treatment. The measurements were conducted in November 2007 (A), February 2008 (B), June 2008 (C), and August 2008 (D). The numbers on the bars are the p-values of t-tests between the two plant types. 
At higher $\mathrm{CO}_{2}$ concentrations, the curve is affected by the reduced ability of the activase to regenerate Ribulose -1, 5-bisphosphate (RuBP) $)^{8)}$.

As shown in Fig. 3, A-Ci curves of PIF1 transgenic and wild-type plants did not show any differences at lower $\mathrm{CO}_{2}$ concentrations, but the $\mathrm{A}-\mathrm{Ci}$ curves of PIF1 transgenic plants decreased non-linearly as $\mathrm{CO}_{2}$ concentration increased. This demonstrates the limitation in the ability to regenerate RuBP. While there was no difference in stomatal conductance between PIF1 transgenic and wild-type plants, the RuBP regeneration capability of wild-type plants was greater than that of transgenic plants.

When we examined photosynthetic responses correlated with PAR in wild-type and cold-stress tolerant tobacco plants (Fig. 4), both plant types showed very similar light saturation points of $1000 \mathrm{~mol} \mathrm{~m}^{-2} \mathrm{~s}^{-1}$ and light compensation points of $20 \sim 50 \mathrm{~mol} \mathrm{~m}^{-2} \mathrm{~s}^{-1}$. Based on

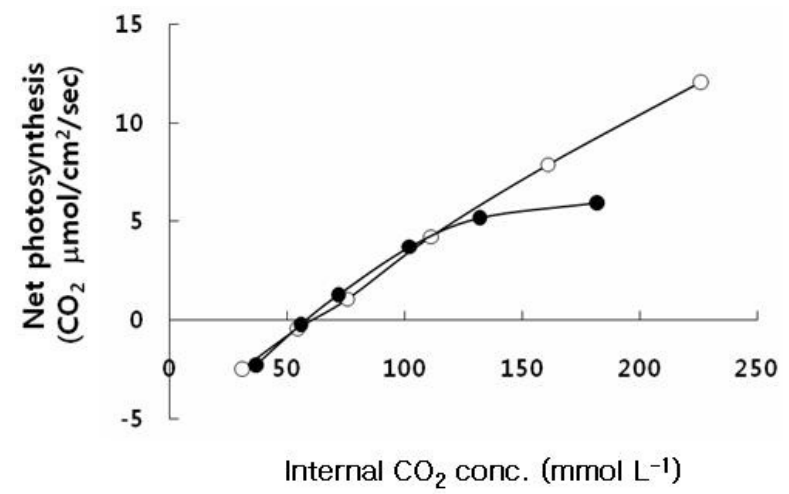

Fig. 3. Photosynthetic responses to variation in internal $\mathrm{CO}_{2}$ concentrations in wild-type $(O)$ and cold-stress tolerant (O) tobacco plants. The supplied $\mathrm{CO}_{2}$ levels in the cuvette were $400,300,200,100,50,0 \mathrm{mmol} \mathrm{L}^{-1}$.

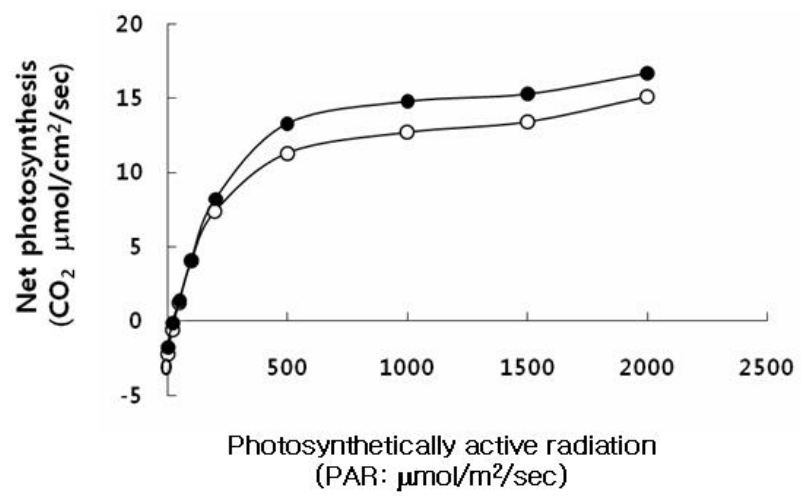

Fig. 4. Photosynthetic responses to variation in photosynthetically active radiation (PAR) in wild-type $(O)$ and cold-stress tolerant $(O)$ tobacco plants. The levels of PAR were 2000, $1500,1000,500,200,100,50,20,0 \mathrm{mmol} \mathrm{m}^{-2} \mathrm{~s}^{-1}$. these results, we estimate that there may be no difference in the light reactions of photosynthesis between the two plant types. From these two additional experiments, we were unable to conclude that overexpression of the PIF1 zinc finger protein makes any ecophysiological difference that leads to coldstress tolerance.

Abiotic stress-inducible zinc finger proteins, which are known to function in regulation of transcription, have been isolated from many plants including soybean (SCOF-1) $)^{9)}$, Arabidopsis (STZ) $)^{10)}$, and rice $\left(\mathrm{OSISAP}^{11)}\right.$ and ZFP245 ${ }^{12)}$ ). We do not know why the gas-exchange ability of the PIF1 transgenic plants is generally lower than that of wild-type plants during the actively growing developmental stages, although the observed differences are not statistically significant.

While PIF1 transgenic tobacco plants had the ability to tolerate cold temperatures, they have lower Cs and Pnet under normal growth temperatures compared to wild-type plants. This implies that, under normal conditions, cold tolerance may not be necessary in terms of evolutionary fitness. Many proteins that are required for cold tolerance are very useful for low-temperature tolerance, but, may not be needed for survival under normal conditions.

\section{Acknowledgement}

This study was partially carried out with the support of Research Cooperating Program for Agricultural Science \& Technology Development (Project No. 20080601036018), RDA, Republic of Korea.

\section{References}

1. Xiong, L, Schumaker, K. S., and Zhu, J. K. (2002) Cell signaling during cold, drought, and salt stress, Plant Cell 14, S165-S183.

2. Seki, M., Narusaka, M., Abe, H., Kasuga, M., Yamaguchi, K., Carninchi, P., Hayashizaki, Y. and Shinozaki, K. (2001) Monitoring the expression pattern of 1300 Arabidopsis genes under drought and cold stresses by using a full-length cDNA microarray, Plant Cell 13, 61-72.

3. Zhu, T. and Provart, N. J. (2003) Transcriptional responoses to low temperature and their regulation in Arabidopsis, Can. J. Bot. 81, 1168-1174. 
4. Thomashow, M. F. (1999) Plant cold acclimation: Freezing tolerance genes and regulatory mechanism, Annu. Rev. Plant Physiol. Plant Mol. Biol. 50, 571-599.

5. Qiang, L, Nanming, Z, Yamaguchi-Shinozaki, K. and Shinozaki, K. (2000) Regulatory role of DREB transcription factors in plant drought, salt and cold tolerance, Chinese Sci. Bull. 45, 970-975.

6. Hwang, E. W., Kim, K. A., Park, S. C., Jeong, M. J., Byun, M. O. and Kwon, H. B. (2005) Expression profiles of hot pepper (Capsicum annuum) genes under cold stress condition, J. Biosci. 30, 657-667.

7. Hwang, E. W., Park, S. C., Byun, M. O., Choi, M., and Kwon, H. B. (2008) Overexpressing of zinc finger protein of Capsicum annuum (PIF1) in tobacco enhances cold tolerance, Genes $\mathcal{E}$ Genomics. 30, 93-99.

8. Hopkins, W. G. and Huner, N. P. A. (2004) Plant physiology, John Wiley \& Sons, Inc., Hoboken, New Jersey, USA, p. 187-198.

9. Kim, J. C., Lee, S. H., Cheong, Y. H., Yoo, C. M., Lee, S. I., Chun, H. J., Yun, D. J., Hong, J. C., Lee, S.
Y., Lim, C. O. and Cho, M. J. (2001) A novel cold-inducible zinc finger protein from soybean, SCOF-1, enhances cold tolerance in transgenic plants. Plant Journal 25(3), 247-259.

10. Sakamoto, H., Maruyama K, Sakuma, Y., Meshi, T., Iwabuchi, M, Shinozaki, K. and Yamaguchi-Shinozaki, K. (2004) Arabidopsis Cys2/His2-type zinc-finger proteins function as transcription repressors under drought, cold, and high-salinity stress conditions, Plant Physiol. 136, 2734-2746.

11. Mukhopadhyay, A., Vij, S. and Tyagi, A. K. (2004) Overexpression of a zinc-finger protein gene from rice confers tolerance to cold, dehydration, and salt stress in transgenic tobacco, Proc. Acad. Sci. USA 101, 6309-6314.

12. Huang, J., Wang, J. F., Wang, Q. H. and Zhang, H. S. (2005) Identification of a rice zinc finger protein whose expression is transiently induced by drought, cold but not by salinity and abscisic acid, DNA Seq., 16, 130-136. 